\title{
Die Verantwortung des Journalisten
}

\author{
Eine Herausforderung für die Journalistenausbildung ${ }^{1}$
}

Walter Hömberg ${ }^{2}$

Am Anfang soll ein Zitat stehen: „Die Verantwortung des Journalisten in einem freiheitlichen, demokratisch verfaßten Staat wie der Bundesrepublik Deutschland hat ihren Grund in der herausgehobenen Funktion (,öffentliche Aufgabe'), die der Journalist durch seine berufliche Tätigkeit im Prozeß der gesellschaftlichen Kommunikation ausübt, indem er als Vermittler an die Öffentlichkeit eine soziale Dienstleistung bewirkt (oder unerfüllt läßt), sei es als Journalist in der Beförderung des Austausches von Nachrichten, Meinungen, Zielvorstellungen usw., sei es als Publizist in der Vertretung von Interessen und Werten (seiner Gruppe, Kirche, Partei usw.)“ (Roegele 1977, S. 207).

Mit diesem Bandwurmsatz beginnt ein Beitrag zum Thema "Verantwortung des Journalisten“, den Otto Bernhard Roegele 1977 im Lexikon „Medienrecht" veröffentlicht hat. Die Form dieses Satzes ist untypisch für den Verfasser. Er favorisiert sonst eine übersichtliche Syntax und einen flüssigen Stil, in dem auch komplexe Sachverhalte klar und verständlich vermittelt werden. Die Form orientiert sich hier wohl primär an den Erwartungserwartungen der Textsorte Lexikonartikel mit ihrem Kondensierungszwang.

Typisch für den Verfasser sind allerdings die Argumentationsweise und der Inhalt der dann folgenden acht Druckseiten, die deshalb die Folie meines Vortrags bilden sollen. Als zentrale Funktion des Journalismus wird die Vermittlung herausgestellt. "Ihr erster Zweck" - so Roegele - „ist die Benachrichtigung der Zeitgenossen über das, was in der Welt vorgeht, über die Probleme, die für sie von Belang sind, über die Möglichkeiten, die zur Lösung dieser Probleme existieren, über die meist gegensätzlichen Meinungen, die darüber geäußert werden und über die Ursachen, Zusammenhänge, Bedingungen, Folgen (...), die dabei zu berücksichtigen sind. Dazu kommt als zweiter nicht minder wichtiger Zweck die möglichst zuverlässige Besorgung des wechselseitigen Austauschs von Ideen, Meinungen, Interessen und Zielvorstellungen, die von einzelnen Gruppen, Verbänden und Institutionen verlautbart werden und in das ,Zeitgespräch der Gesellschaft' einzubringen sind“ (ebd., S. 208). Hier wird der archimedische Punkt der so genannten Münchner Schule angesprochen und aufgegriffen: Aktuelle öffentliche Kommunikation, das ist "Zeitgespräch der Gesellschaft". Die öffentliche Aufgabe des Journalisten liegt darin, dieses "Gespräch“ zu moderieren, das heißt die unterschiedlichen Positionen zu Wort kommen zu lassen (vgl. Aswerus 1993).

Der Artikel 5 des Grundgesetzes garantiert Meinungsäußerungsfreiheit, Meinungsverbreitungsfreiheit und Informationsfreiheit. Dabei handelt es sich nicht um professi-

1 Vortrag auf dem Symposium der Sozialwissenschaftlichen Fakultät zu Ehren von Otto B. Roegele am 27. Januar 2006 an der Universität München.

2 Walter Hömberg ist Professor für Journalistik an der Katholischen Universität Eichstätt.

Elektronische Publikationen der Universität München. Kommunikations- und Medienforschung. Münchener Beiträge zur Kommunikationswissenschaft Nr. 5, Mai 2006. URL:

http://epub.ub.uni-muenchen.de/archive/00000936 
onelle Sonderrechte für bestimmte Berufe, sondern um allgemeine Bürgerrechte. Die Kommunikationsfreiheit ist ein "Jedermannsrecht", konstitutiv für die Demokratie. Ebenso plausibel wie die demokratietheoretische Begründung der allgemeinen Informations- und Kommunikationsfreiheit ist freilich die Erkenntnis, dass der Journalist eine "Schlüsselposition im Netzwerk des Kommunikationsgeschehens" einnimmt (Roegele 1977, S. 208). Aus dieser Privilegisierung resultiert eine besondere Verantwortung. Roegele postuliert: „Weil er von Berufs wegen darauf spezialisiert ist und weil ihm im Verfolg der Arbeitsteilung die Besorgung des Geschäfts der öffentlichen Kommunikation übertragen wurde, ist er verpflichtet, sein Bestes zu tun, um Information, Orientierung und öffentliche Debatte im Vorfeld der Entscheidungen zustandezubringen. Die Verantwortung des Journalisten muß umso größer werden, je mehr der Anteil an wichtigen Kenntnissen, die der Staatsbürger nur indirekt, durch die Vermittlung von Massenmedien, erfahren kann, wächst, und je geringer der Anteil der durch direkte Wahrnehmung gewonnenen Kenntnisse wird, zumal dabei auch die Möglichkeit der Überprüfung der vermittelten Informationen mit Hilfe der unmittelbar erfahrenen schwindet" (ebd.).

In wenigen Strichen hat der Autor zuvor die historische Entwicklung von kleinräumigen Verhältnissen, in denen mündliche Benachrichtigung und Diskussion als Kommunikationsformen ausreichten, $\mathrm{zu}$ den modernen Flächenstaaten nachgezeichnet. Anders als in den Stadtrepubliken der Antike oder den Urkantonen der Schweiz reichen Agora, Forum und Landsgemeinde als Orte der Verständigung gegenwärtig nicht mehr aus. Vielmehr bedarf es heute der Massenmedien, um die notwendigen Kommunikationsleistungen zu erbringen. Hier blitzt kurz auf, was für viele der Schriften Roegeles charakteristisch ist. Imponierend die Weite seines Blicks. Das gilt zunächst in zeitlicher Hinsicht. Altertum, Mittelalter, Neuzeit sind für den gelernten Historiker ${ }^{3}$ mehr als nur ein Fundus für Ereignisse und Erzählungen. Geschichte ist vielmehr - im Sinne des Theologen Hans von Keler - „nicht nur Geschehenes, sondern Geschichtetes - also der Boden, auf dem wir stehen und bauen" (vgl. Hömberg 2005, S. 188). Vergangenheit, Gegenwart, Zukunft - alles ist da, häufig in Simultanpräsenz.

Auch auf das Glatteis der Prognose hat sich dieser Autor gewagt. Mit Prognosen ist es bekanntlich so eine Sache - besonders, wenn sie in die Zukunft gerichtet sind. Im Jahre 1970 hat Otto B. Roegele ein schmales Bändchen mit dem Titel „Die Zukunft der Massenmedien" veröffentlicht (Roegele 1970). Ausgehend vom "Siegeszug des Fernsehens" diskutiert er darin die Potenziale der Kommunikations-Satelliten, des KabelFernsehens und der Bild-Ton-Kassetten. Anders als die Berge von prognostischer Literatur, die in den folgenden Jahrzehnten aufgehäuft wurden (vgl. exemplarisch Magnus 1975) und häufig nichts anderes als Prognosemüll produzierten, verzichtet der Verfasser hier auf Spekulationen und Visionen. Er schlägt sich weder auf die Seite der Apologeten, die die neuen technischen Möglichkeiten und ökonomischen Vorteile der expandierenden Telekommunikation herausstellen, noch auf die Seite der Apokalyptiker, die eindringlich vor den gesellschaftlichen Folgen warnen, vor Kulturverfall, Desintegration, rückläufigen Sozialbeziehungen. Computer und Internet waren damals noch nicht einmal ein Thema für die Science-Fiction-Branche. Aber der Autor thematisiert schon die Informationsflut: „Wer im Hochwasser ertrinkt, ist (...) genauso tot wie ein anderer, der infolge Wassermangels verdurstet" (Roegele 1970, S. 51). Seine Reflexionen über die Zukunft der Presse, die zunehmende Medienkonzentration und die Internationalisierung des Medienmarktes sind zwar nicht spektakulär, aber vielleicht gera-

3 Otto B. Roegele studierte von 1938 bis 1945 Philosophie, Geschichte und Medizin. 
de deshalb noch heute lesenswert - zumal in diesen Tagen, wo wieder eine Elefantenhochzeit ansteht. ${ }^{4}$

Die Weite des Blicks - das gilt auch für die räumliche Dimension. Mit vielen Ländern und Kulturen Europas hat sich Roegele intensiv befasst, vor allem mit Frankreich - gemeinsame Seminare für deutsche und französische Journalisten gehen auf seine Initiative zurück -, weiter mit Ländern wie Italien, Spanien, Polen und Jugoslawien. Ich erinnere mich an ein gemeinsames interdisziplinäres Seminar vor drei Jahrzehnten in Dubrovnik, bei dem Lust und Nutz in schöner Balance standen. Dazu kam sein Engagement in internationalen Organisationen wie der AIERI ${ }^{5}$ und der UNESCO. Internationalität und Interdisziplinarität - diese Schlagwörter aus dem verbalen Notfallkoffer der permanenten Curriculumreform, hier waren sie in einer Person verkörpert.

Zurück zum Thema Verantwortung des Journalisten. Die bisher zitierten Passagen aus Roegeles Aufsatz setzen an beim journalistischen Individuum. Neben der Mikroebene nimmt er in einer erweiterten Fassung aus dem Jahre 1994 auch die Meso- und die Makroebene ins Visier (Roegele 1994). Journalisten arbeiten in Organisationen, konkret: in Redaktionen. Der Verfasser dazu: „Zeitungen und Zeitschriften, Radiound Fernsehprogramme sind in der Regel die Ergebnisse des Zusammenwirkens einer großen Zahl von Menschen, das bestimmten betrieblichen Organisationsmustern unterworfen ist und dem einzelnen nur einen mehr oder weniger begrenzten Freiraum eigener Entscheidungen überläßt" (ebd., S. 429). Die nächste Ebene ist durch die staatliche und gesellschaftliche Grundordnung bestimmt. Demokratisch-pluralistische, autoritäre und totalitäre Systeme haben ganz unterschiedliche Vorstellungen von der Rolle des Journalisten im Prozess der gesellschaftlichen Kommunikation.

In den vergangenen vier Jahrzehnten hat sich in unserem Fach die Systemtheorie weitgehend durchgesetzt (vgl. Luhmann 1984, Rühl 1969, Löffelholz 2004). Sie liefert uns einen analytischen Generalschlüssel für kommunikative Prozesse auf verschiedenen Ebenen: auf der Interaktions-, der Organisations- und der Gesellschaftsebene. Als umweltbezogener Ansatz hilft sie, Dependenzen und Interdependenzen zwischen unterschiedlichen Systemen und Subsystemen aufzuspüren und zu erklären. Außerdem ist sie anschlussfähig für empirische Forschung.

Probleme macht indes die Ausblendung des Subjektbegriffs beziehungsweise die Ablehnung des Begriffs der Person. Ulrich Saxer schreibt: „Mit der Konzeption von Personen als psychischen Systemen ermöglicht die Systemtheorie in erster Linie, das Feld der kommunikationswissenschaftlichen Forschungsgegenstände $\mathrm{zu}$ homogenisieren und erleichtert damit dessen integrale Bearbeitung. Von Luhmanns Theorie der Sozialsysteme aus bilden Individuen Umwelt von sozialen Systemen, da die Mitgliedschaft in solchen gewöhnlich nicht ihre volle Persönlichkeit beansprucht" (Saxer 2004, S. 87f.). Theorieimmanent mag das überzeugen. Die problematischen Seiten zeigen sich jedoch spätestens bei der Umsetzung auf Felder wie Kommunikationsrecht und Kommunikationsethik. Zwar gibt es auch hier manchmal organisatorische Lösungen - wie etwa beim so genannten "Sitzredakteur“, der nach dem Reichspressegesetz von 1874 stellvertretend für die Redaktion ins Gefängnis ging. Aber dort saß er dann als ganze Person - auch alle seine sonstigen „Rollen“ musste er mitnehmen (vgl. Wetzel 1982, S. 137).

Für Roegele jedenfalls steht fest: Bei allen organisations- und systembedingten Parametern - die Verantwortung für das eigene berufliche Handeln bleibt letztlich beim Individuum. Die „normative Entkernung des Journalismus“, die angesichts der neue-

4 Im Sommer 2005 äußerte der Axel-Springer-Konzern Interesse an der Übernahme der ProSiebenSat.1 Media AG. Sowohl das Kartellamt als auch die Kommission zur Ermittlung der Konzentration im Medienbereich (KEK) lehnten die Übernahme ab (Der Spiegel Nr. 6/2006).

5 Association Internationale des Etudes et Recherches sur l'Information et la Communication. 
ren Berufsforschung diagnostiziert wurde - bei diesem Autor findet sie nicht statt. Mit Bezug auf einschlägige medienbezogene Richtlinien und berufsbezogene Kodizes erläutert er die wichtigsten Berufsnormen. Da sind zum einen Gebote wie Sorgfaltspflicht, Pflicht zur Erforschung des Wahrheitsgehalts und Persönlichkeitsschutz, zum anderen Verbote wie Diskriminierung nationaler, rassischer oder weltanschaulicher Gruppen sowie - wieder ganz aktuell - Schleichwerbung (Roegele 1994, S. 433f.). Auch der dritte Bereich der Kommunikationsethik ist thematisiert: die Publikumsethik. Roegele verwendet diesen Begriff nicht, zeigt aber am Beispiel von Gewaltdarstellungen in Film und Fernsehen und am Anziehen der Reizschraube als Folge zunehmender Medienkonkurrenz im dualen Rundfunksystem gesellschaftliche Gefährdungen auf. Publikum und Programmmacher haben hier gemeinsam Verantwortung (ebd., S. 434f.).

Die enge Verbindung von Journalisten und Publikum hat Otto B. Roegele in seinem Buch „Neugier als Laster und Tugend“ unterstrichen (Roegele 1982). Die Neugier hat keinen guten Ruf. Zwar finden die Enthüllungen der Schlüssellochpresse und die Voyeurshows im Fernsehen allemal ihr Publikum - aber kaum jemand mag zugeben, dass er dazu gehört. Die Neugier, lateinisch curiositas, hat allerdings auch eine andere Seite: Die Entdeckungslust des Forschers, das Informationsbedürfnis des Staatsbürgers, der Wissensdurst überhaupt - seit der Aufklärung gelten sie als Urtrieb des Menschen (ebd., S. 7-18). Nun ist die Aufmerksamkeit sowohl des Einzelnen als auch der Gesellschaft insgesamt ein knappes Gut (vgl. Meyen 2004, S. 117ff.). Zur öffentlichen Aufgabe der Medien gehört es, die Neugier-Erwartungen ihrer Nutzer zu erfüllen. Neugier ist der Motor journalistischer Recherche (Roegele 1982, S. 19ff.). Egon Erwin Kisch, der sich ja selbst zum „rasenden Reporter“ stilisierte, hat dies sehr plastisch beschrieben: „Ich kann in keiner Straßenbahn fahren, ohne herauskriegen zu wollen, welches Buch der Herr in der entgegengesetzten Ecke liest. Ich verfolge ein Paar durch mehrere Straßen, um zu erfahren, welche Sprache sie sprechen. Ich gaffe in fremde Fenster, ich lese alle Wohnungsschilder in dem Haus, in dem ich zu Besuch bin, ich durchforsche Friedhöfe nach vertrauten Namen. Gleichgültige Menschen frage ich über ihr Leben aus. Ungewöhnliche Straßenbezeichnungen zwingen mich, zu ergründen, warum sie so lauten. Jede Rumpelkammer und jeden Stoß alter Papiere möchte ich durchsuchen, jedes ,Eintritt verboten' lockt mich zum Eintritt, jede Geheimhaltung zur Nachforschung" (Kisch 1993, S. 73).

Otto B. Roegele hat in seinem Neugier-Buch von 1982 nicht nur gezeigt, dass Aufmerksamkeit das Kommunikationssystem steuert und damit zentrale Thesen von Georg Francks "Ökonomie der Aufmerksamkeit" vorweg genommen, sondern er hat darüber hinaus die Journalisten treffend als Auftragsverwalter der Neugier charakterisiert (vgl. Franck 1998).

Vor gut fünf Jahren hat Angela Merkel dem „SZ-Magazin“ ein langes Interview gegeben. Die 36. von 100 Fragen lautete: „Eine typische Politikervokabel, die Thnen nicht über die Lippen kommt?“ Die CDU-Vorsitzende antwortete: „Möglichst selten ,Herausforderung. ${ }^{\prime \prime 6}$ Nun steht diese „typische Politikervokabel“ ausgerechnet im Untertitel meines Vortrags. Ich will es kurz machen, und greife dabei wieder auf ein Zitat von Otto B. Roegele zurück. Am Ende seiner Schrift über „Die Zukunft der Massenmedien“ resümiert er: „Wie Leistungen und Bedeutung des Kommunikationswesens in der Gesellschaft der Zukunft wachsen werden, so wird auch die Zahl der in Kommunikationsberufen Tätigen zunehmen - und damit wird sich die Frage nach deren Verantwortung, Ausbildung und Weiterbildung mit neuer Dringlichkeit stellen“ (Roegele 1970, S. 52). Dies schreibt jemand, der die zwei Berufe, die sein Lebenswerk ausmachen, jeweils als Seiteneinsteiger begonnen hat. Journalist wurde er, weil der schwer erkrankte

6 Wieder abgedruckt im SZ-Magazin, Nr. 47 vom 25. November 2005, S. 24-27, hier S. 26. 
Gründer des „Rheinischen Merkurs“7 den jungen Arzt, der ihm als freier Mitarbeiter aufgefallen war, 1948 als Leiter des Ressorts Kulturpolitik in die Redaktion holte. Bereits im Jahr darauf war er Chefredakteur des Blattes, und er blieb es während der gesamten Regierungszeit Adenauers, dessen Politik er begleitete - reflektierend und wohl auch inspirierend. 1963 folgte er dem Ruf auf den traditionsreichen Lehrstuhl für Zeitungswissenschaft an der Universität München. Professor wurde er - und zugleich Student: Zeitungswissenschaft hatte er ja nie studiert. Als Professor holte er dieses Studium nach - sehr erfolgreich, wie wir wissen.

Vielleicht waren es gerade diese Erfahrungen als Seiteneinsteiger, die ihn dazu brachten, den neuen Studiengang Journalistik vehement zu unterstützen. Der Diplomstudiengang mit seiner Integration von theoretischer und praktischer Ausbildung, dem integrierten sozialwissenschaftlichen Grundstudium und dem dezidierten Berufsbezug war eine wirkliche Innovation. ${ }^{8}$ Kann man Verantwortung und Verantwortlichkeit lehren und lernen? Sicher nicht, indem man ein Ausbildungsmodul entsprechend etikettiert. Aber man kann, man muss die Grundprinzipien und zugleich die Fallen der Ethik des Journalismus und anderer Kommunikationsberufe im Studium kennen lernen. Die Vorstellung, dass sich von der Ausbildungs-Wurzel alle Defizite unseres Mediensystems kurieren lassen, hat sich als illusionär erwiesen. Aber dass entsprechende Studiengänge die Professionalität und die Qualität der Medienpraxis verbessern können, das zeigen die vielen erfolgreichen Absolventen dieses Instituts und der anderen Journalistik-Studiengänge.

Es war ein Leben im Spagat: Auch als Wissenschaftler hat Roegele seinem Blatt, dem „Rheinischen Merkur", die Treue gehalten - als Herausgeber und Leitartikler, als Berichterstatter und Kritiker. Der Wissenschaftler, der die „Neophilie“ der Massenmedien oft genug analysiert hat (Roegele 2000, S. 55ff.), wusste um die Trendwellen und Themenkonjunkturen des "Zeitgesprächs der Gesellschaft". Der Publizist hat immer wieder versucht gegenzuhalten: alteuropäisch gebildet, wertebewusst, Zeitgeisttabus souverän missachtend - und dabei tolerant gegenüber anderen Denk- und Lebensstilen. Sein Sohn Bernhard hat in einem Brief an mich die Tätigkeit seines Vaters in München treffend charakterisiert: Sie war weniger verbunden „mit der Konstruktion eines Lehrgebäudes als mit der Errichtung und dem Betrieb eines Forums, auf dem Theorie, Empirie, Geschichte und Praxis sich begegnen und Menschen voneinander lernen können". Den Ertrag habe er an ihrem Lebens- und Berufsweg festgemacht.

Otto B. Roegele hat keine „Memoiren“ im emphatischen Sinne geschrieben. Aber er hat in mehreren Aufsätzen über die wichtigsten Etappen seines Lebens berichtet (Roegele 1966, 1988, 1997). Am Ende seiner Darstellung über die Jahre in München schreibt er, dass er gerne mit den längst verstorbenen Gründern des Münchner Instituts über seine Erfahrungen diskutieren würde (Roegele 1997, S. 108). Ich kann mir noch manche andere Gesprächspartner im „Reiche derer Todten“ für ihn vorstellen. ${ }^{9}$ Joseph von Görres und Karl Marx, über die er seine ersten Lehrveranstaltungen gehalten hat, sind gewiss darunter. Und dazu gehören nun leider auch Peter Glotz und Heinz Starkulla. ${ }^{10}$

7 Franz Albert Kramer (1900 bis 1950). Katholisch-konservativer Publizist. 1946 Gründung des "Rheinischen Merkur".

8 Der Diplomstudiengang Journalistik, wurde 1974 als Kooperation zwischen der Deutschen Journalistenschule und dem Institut für Kommunikationswissenschaft (Zeitungswissenschaft) als Modellversuch genehmigt und 2003 eingestellt (vgl. Höfler 2005, S. 103).

9 Die an ältere Vorbilder anschließende Zeitschrift „Gespräche in dem Reiche der Todten“ von David Faßmann erschien zwischen 1718 und 1739. Von 1786 bis 1810 brachte Moritz Flavius Trenck von Tonder „Politische Gespräche im Reiche der Todten“ heraus.

10 Peter Glotz, geboren am 6. März 1939, gestorben am 25. August 2005. Heinz Starkulla, geboren am 4. Oktober 1922, gestorben am 25. November 2005. 


\section{Literatur}

Aswerus, Bernd M. (1993): Vom Zeitgespräch der Gesellschaft. Zusammengestellt und eingeführt von Hans Wagner. München: Fischer

Franck, Georg (1998): Ökonomie der Aufmerksamkeit. Ein Entwurf. München: Hanser

Kisch, Egon Erwin (1993): Vorträge und Theater. In: Ders.: Marktplatz der Sensationen. 6. Auflage. Berlin, Weimar: Aufbau Verlag, S. 62-77

Höfler, Barbara (2005): Ende der Debatte? Bilanz der Erfahrungen aus 30 Jahren „Münchener Modell“ am Institut für Kommunikationswissenschaft und Medienkunde. Eine Fachgeschichte. München (Diplomarbeit)

Hömberg, Walter (2005): Brauchen wir noch Journalisten? Ein Blick zurück nach vorn. In: Jäckel, Michael/Haase, Frank (Hrsg.): In medias res: Herausforderung Informationsgesellschaft. München: Kopaed, S. 187-204

Löffelholz, Martin (2004) (Hrsg.): Theorien des Journalismus. Ein diskursives Handbuch. 2. Auflage. Wiesbaden: VS Verlag für Sozialwissenschaften

Luhmann, Niklas (1984): Soziale Systeme. Grundriß einer allgemeinen Theorie. Frankfurt am Main: Suhrkamp

Magnus, Uwe (1975) (Hrsg.): Massenmedien in der Prognose. Konzepte und Modelle für die Zukunft. 2. Auflage. Berlin: Spiess

Meyen, Michael (2004): Mediennutzung. Mediaforschung, Medienfunktionen, Nutzungsmuster. 2. Auflage. Konstanz: UVK

Roegele, Otto B. (1966): Student im Dritten Reich. In: Kuhn, Helmut: Die deutsche Universität im Dritten Reich. Acht Beiträge. München: Piper, S. 135-174

Roegele, Otto B. (1970): Die Zukunft der Massenmedien. Osnabrück: Fromm

Roegele, Otto B. (1977): Verantwortung des Journalisten. In: Schiwy, Peter/Schütz, Walter J. (Hrsg.): Medienrecht. Stichwörter für die Praxis. Neuwied, Darmstadt: Luchterhand, S. 207-215

Roegele, Otto B. (1982): Neugier als Laster und Tugend. Zürich: Edition Interfrom

Roegele, Otto B. (1988): Schüler im „Dritten Reich“. Ein autobiographisches Fragment. In: Weinacht, Paul Ludwig (Hrsg.): Gelb-rot-gelbe Regierungsjahre. Badische Politik nach 1945. Gedenkschrift zum 100. Geburtstag Leo Wohlebs (1888-1955). Sigmaringendorf: Regio, S. 23-32

Roegele, Otto B. (1994): Verantwortung des Journalisten. In: Schiwy, Peter/Schütz, Walter J. (Hrsg.): Medienrecht. Lexikon für Wissenschaft und Praxis. 3. Auflage. Neuwied: Luchterhand, S. 427-439

Roegele, Otto B. (1997): Ausbreitung, Lähmung, Konsolidierung - München 1963-1985. In: Kutsch, Arnulf/Pöttker, Horst (Hrsg.): Kommunikationswissenschaft - autobiographisch. Zur Entwicklung einer Wissenschaft in Deutschland. Wiesbaden: Westdeutscher Verlag, S. 62-109

Roegele, Otto B. (2000): Massenmedien und Regierbarkeit. In: Ders.: Plädoyer für Publizistische Verantwortung. Beiträge zu Journalismus, Medien und Kommunikation. Herausgegeben von Petra Dorsch-Jungsberger, Walter Hömberg und Walter J. Schütz. Konstanz: UVK, S. 51-77

Rühl, Manfred (1969): Die Zeitungsredaktion als organisiertes soziales System. Bielefeld: Bertelsmann

Saxer, Ulrich (2004): Systemtheorie und Kommunikationswissenschaft. In: Burkart, Roland/Hömberg, Walter (Hrsg.): Kommunikationstheorien. Ein Textbuch zur Einführung. 3. Auflage. Wien: Braumüller, S. 85-110

Wetzel, Hans-Wolfgang (1982): Kulturkampf-Gesetzgebung und Sozialistengesetz (1871/74-1890). In: Fischer, Heinz-Dietrich (Hrsg.): Deutsche Kommunikationskontrolle des 15. bis 20. Jahrhunderts. München u.a.: Saur, S. 131-152 